\title{
Haben Langschläfer ein erhöhtes Schlaganfallrisiko?
}

\author{
Studienergebnisse weisen darauf hin, dass der Schlaf einen \\ Prädiktor für zukünftige kardiovaskuläre Ereignisse darstellt. \\ Von besonderer Bedeutung ist in diesem Zusammenhang das \\ Auftreten von Schlaganfällen. Für Großbritannien fehlen \\ derzeit entsprechende Daten. Aus diesem Grund haben Y. Leng \\ et al. innerhalb einer britischen Population die mögliche \\ Assoziation zwischen der Schlafdauer und dem Risiko für \\ Schlaganfall untersucht. \\ Neurology 2015; 84: 1072-1079
}

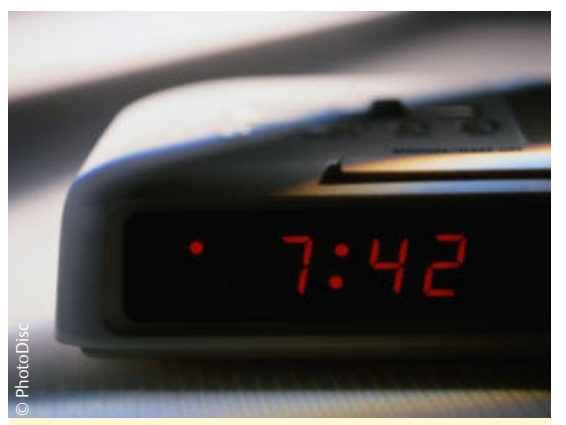

Als Normalschläfer gilt, wer 7-9 Stunden schläft. Manche Menschen kommen mit weniger Schlaf aus, andere benötigen mehr. Diese Unterschiede sind häufig genetisch bedingt.

Die prospektive Studie berücksichtigte 9692 schlaganfallfreie Personen im Alter von 42-81 Jahren. Diese waren Teilnehmer der European Prospective Investigation into Cancer Norfolk Studie. Für die Zeiträume 1998-2000 und 2002-2004 wurde jeweils die Schlafdauer erfasst sowie die bis zum 31. März 2009 aufgetretenen Schlaganfälle. Zudem identifizierten die Autoren innerhalb der Datenbanken Ovid Medline, EMBASE und Cochrane Library Studien, die die Assoziation zwischen der Schlafdauer und der Schlaganfallinzidenz zum Inhalt hatten und bis Mai 2014 publiziert wurden. Diese Daten waren Grundlage für eine Metaanalyse.

Das Durchschnittsalter der Studienteilnehmer betrug 61,6 Jahre. 69\% gaben an, zwischen 6 und 8 Stunden pro Tag zu schlafen, bei $10 \%$ betrug die tägliche Schlafdauer $>8$ Stunden. Während der im Durchschnitt 9,5 Jahre dauernden Nachbeobachtung erlitten 346 Personen min-

destens einen Schlaganfall. Ein langer Schlaf war nach Adjustierung für alle Kovariate signifikant mit einem erhöhten Schlaganfallrisiko assoziiert (Hazard Ratio $[H R]=1,46$ ). Die Assoziation blieb auch bei Personen ohne vorbestehende Krankheiten und bei solchen mit gutem Schlaf stabil. Auch im Fall von Individuen mit kürzerem Schlaf bestand ein erhöhtes Risiko für einen Schlaganfall $(H R=1,18)$, allerdings war das Ergebnis statistisch nicht signifikant.

Die Autoren identifizierten ein höheres Schlaganfallrisiko für Personen mit einem anhaltend langen Schlaf bzw. einer deutlichen Zunahme der Schlafdauer über den Zeitverlauf gegenüber solchen mit einem anhaltend durchschnittlichen Schlaf. In die Metaanalyse gingen Daten aus 11 Studien ein (559252 Teilnehmer aus 7 Ländern). Im Fall eines kurzen Schlafs war das gepoolte relative Risiko 1,15 und im Fall eines langen Schlafs 1,45.

\begin{abstract}
Fazit
Innerhalb der prospektiven Studie und Metaanalyse war ein längerer Schlaf mit einem erhöhten Risiko für einen späteren Schlaganfall assoziiert. Ein zwar vorhandener, aber weniger stark ausgeprägter Zusammenhang bestand zwischen einer kürzeren Schlafdauer und dem Schlaganfallrisiko. Die Autoren empfehlen, den zugrundeliegenden Mechanismus genauer zu untersuchen.
\end{abstract}

Dr. Frank Lichert, Weilburg
Morbus Alzheimer

\section{Forscher entdecken neues Peptid}

Bei Alzheimer-Kranken sammeln sich Eiweißklumpen im Gehirn an, die die Nervenzellen schädigen. Als Auslöser hierfür gelten ß-Amyloid-Peptide, deren Bildung von der $ß$-Sekretase, einem scherenartigen Enzym, angeregt wird. Nun gelang einem internationalen Forscherteam um Prof. C. Haass und Dr. M. Willem, München, eine fundamentale Entdeckung: „Es gibt einen zweiten Weg, bei dem die scherenartigen Enzyme ein alternatives Eiweiß aus dem Vorläuferprotein herausschneiden“, sagt Haass. Die Forscher gaben dem neu entdeckten Peptid den Namen Amyloid-n. Über ihre Ergebnisse berichten sie in Nature (Nature 2015; DOI: 10.1038/nature14864).

In Zusammenarbeit mit der Neurobiologin Dr. H. Marie, Valbonne/Frankreich, sowie Prof. A. Konnerth und Dr. M. A. Busche, München, gelang es den Wissenschaftlern, die Funktion von Amyloid- $\eta$ im Gehirn zu bestimmen: Während verklumptes ß-Amyloid für Chaos sorgt, weil es Nervenzellen überaktiviert, bremst Amyloid-n die neuronale Stimulation. „Offenbar haben die 2 kleinen Eiweiße, die aus ein- und demselben Vorläuferprotein herausgeschnitten werden, gegensätzliche Wirkungen, die normalerweise genau austariert sind“, sagt Haass.

Diese Entdeckung hat einen direkten Einfluss auf derzeitige therapeutische Studien am Menschen, die sich bislang auf das ß-Amyloid konzentrieren. So wird aktuell untersucht, ob die Unterdrückung der ßSekretase dazu führt, dass sich der Gedächtnisverlust bei Alzheimerpatienten verlangsamt. Die Forscher um Haass und Willem haben nun festgestellt, dass die Blockade der ß-Sekretase zwar zu einer Reduktion von ß-Amyloid führt, aber auch gleichzeitig eine massive Überproduktion von Amyloid- $\eta$ zur Folge hat. „Damit könnte es zu einer Störung der neuronalen Aktivität und damit der Gehirnfunktion kommen“, sagt Haass. Die Wissenschaftler raten daher, solche bisher nicht erwarteten Nebenwirkungen in klinischen Studien genau zu verfolgen.

Nach einer Mitteilung der LudwigMaximilians-Universität München 